\title{
Microwave Detection of Water Pollution in Underground Pipelines
}

\author{
Ahmad H. Abdelgwad ${ }^{\text {a }}$, Tarek M. Said ${ }^{\mathrm{b}}$, Amr M. Gody $^{\mathrm{c}}$ \\ Fayoum University, Faculty of Engineering, Department of Electrical Engineering, Fayoum, POBox: 63514, \\ Egypt
}

\begin{abstract}
An electromagnetic model is proposed to detect water pollution in underground pipelines. Contaminants present above a certain level in water can be a public health hazard. The contrast in the dielectric constant between contaminated and fresh water is one of the most important parameters to be considered for detecting the presence of pollutants in water. Simulations of frequency response and time domain pulse wave through a multi-layer medium are presented. The complex dielectric permittivity of polluted water has been measured as a function of frequency and analytically represented by Cole-Cole fit model. Water pollution can be detected by observing the variation of the reflection coefficient or reflected signals from unpolluted and polluted water. The experimental set up is described and the procedure followed to obtain an effective permittivity data is outlined. These measurements are, to the best of the author's knowledge, the first of its kind to be published. Microwave technique discussed in this manuscript for water pollution study is a pioneer technique to detect various pollutants in water.
\end{abstract}

Index Terms: Remote sensing, water pollution, Cole-Cole model, ground penetrating radar (GPR), reflection coefficient.

(C) 2014 Published by MECS Publisher. Selection and/or peer review under responsibility of the Research Association of Modern Education and Computer Science

\section{Introduction}

Environmental pollution is a major problem in the modern world. It includes water pollution, air pollution and sound pollution. The detection and control of pollutants in water have great importance. Pollutants of water can be organic pollutants, inorganic pollutants, biological pollutants, physiological pollutants and physical pollutants [1]. Recently, many parts of the world are suffering because of a lack of clean water. Contaminants present above a certain level in water are extremely harmful to human beings' health. Water quality intended for human consumption is usually high when it leaves the purification plant, but it can deteriorate on its way to the consumers for the presence of chemical substances. The pollution of water in underground pipelines may be

* Corresponding author.

Email address: 
caused by the interaction of water with pipe material causing some types of algae and bacteria. Densities of fixed bacterial biomass on different pipe materials incubated in drinking waters were measured by Niquette et al. [2]. Water in underground pipelines may be also contaminated by leaks or explosions that may occur in the pipeline, as every leak or explosion is a potential entry point for contaminants hence it create a public health risk [3]. As a result, water monitoring is of primary concern for most health and security authorities. Since water pipes are buried underground, excavation is very expensive. Nowadays, water pollution is detected in laboratories, where small samples of water are analyzed for different contaminants. However, testing for all known water contaminants over a wide area is a complicated and expensive process. Analysis of the available technologies to date shows that conventional wireless sensors do not entirely meet the needs of the users. Currently available multi-sensor systems give only an approximate estimation of the water composition [4]. Though these systems are promising, they are reliable for a short period only and need further research and development. The lack of accurate, cost-effective and robust sensors remains a challenge.

Ground Penetrating Radar (GPR) is one of the most used tools in the field of the water monitoring and management [5]. GPR produces microwaves electromagnetic radiation that propagates through the ground then returns to the surface. It has been very successful in many applications such as the drainage pipes detection and the determination of the time-behavior of water content in the soil, but it has not been applied to the detection of water pollution in underground pipelines [6]. Microwave propagation through obscuring layers is recently of tremendous interests with practical applications in security, inspection, and mine detection [7-11]. The aim of this paper is to demonstrate the feasibility to detect water contaminants by exploiting microwave technology. As well-known, the presence of pollutants in water alters its complex permittivity (i.e. the dielectric permittivity and conductivity). Detection of pollution is based on the contrast in the dielectric constant between contaminated and clean water. Microwave technology represents a promising and cost-effective solution for detecting water contaminants.

The prime objective of this study is to detect water pollution in underground pipelines. This paper presents frequency and time domain simulation models based on a multi-layer medium for water pollution detection in underground pipelines. The dielectric model of the materials of interest has been studied also. Results are obtained in both time and frequency domains, for different values of the water electromagnetic parameters, corresponding to fresh and contaminated water. Two different values of the pipe burial depth and of its diameter are considered. The results show indications that microwave sensing of water pollution in underground pipelines is a novel application. The current study provides a tool to estimate the responses at different frequencies and configurations which is instrumental to detecting of water pollution in underground pipelines.

The paper is organized as follows. Section 2 is devoted at introducing a multilayer electromagnetic model of a pipe buried in sandy soil. In Section 3 we describe the relationship between the Electromagnetic wave model and transmission line model. In Section 4 we present the dielectric modeling of materials of interest for the proposed electromagnetic model. In section 5 we provide some example results achieved by the microwave approach in the time and frequency domain.

\section{Multilayer Electromagnetic Model}

Ground Penetrating Radar (GPR) system is based on a reflection technique which uses high frequency electromagnetic wave to acquire subsurface information. Assuming an electromagnetic (EM) wave propagates from a GPR and paths through different media of different electrical properties, the multi-layer model is shown schematically in Fig. 1 


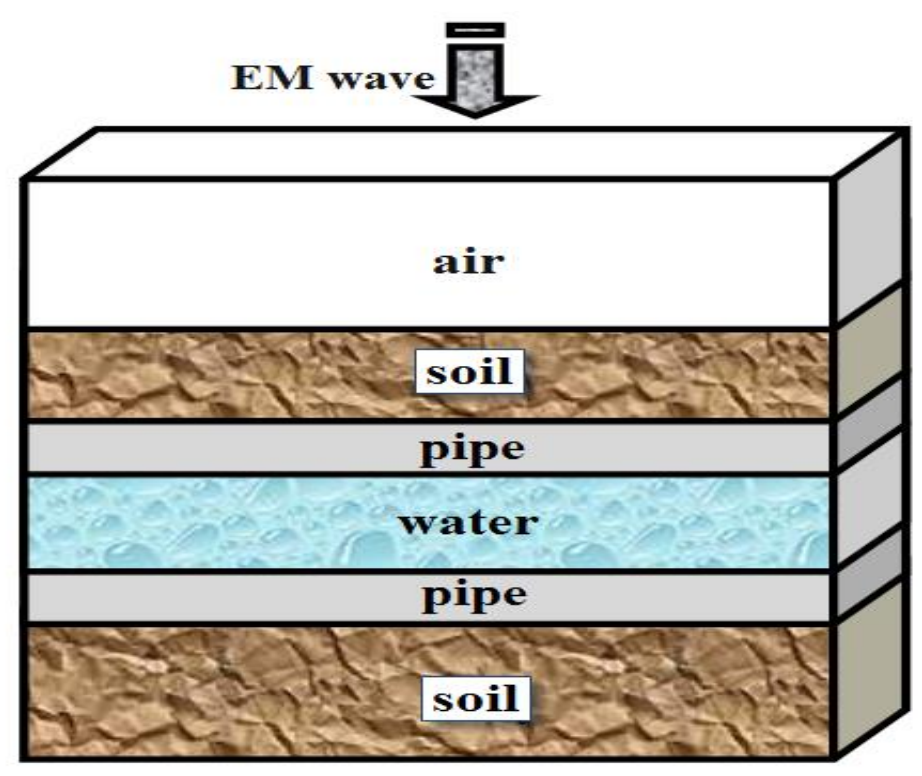

Fig 1. Multi-layer transmission model seen by transmitted EM wave.

When a travelling electromagnetic wave propagates through one medium and enters the other with different electrical properties than the first one, it experiences reflection. At all inter-medium junctures, transmitted wave has a reflection such that at every such junction part of the wave reflects back and the rest moves forward, where multiple reflections within each layer are included. It has the flexibility for more complicated structures to be added, including rough surface interfaces and small inclusions in layers. The equations for the voltage in the transmission line (T.L.) model of this work are identical to Maxwell's equations for GPR problem. Therefore, the results of this paper for solutions to the transmission line voltage are equivalent to the predicted electric field for this problem.

\section{Multilayer Transmission Line Model}

A transmission line model is equivalent to the uniform plane wave propagation model; hence the materials in the multi-layer propagation model illustrated in Fig. 1 are modelled with their characteristic impedances, as shown in Fig. 2.

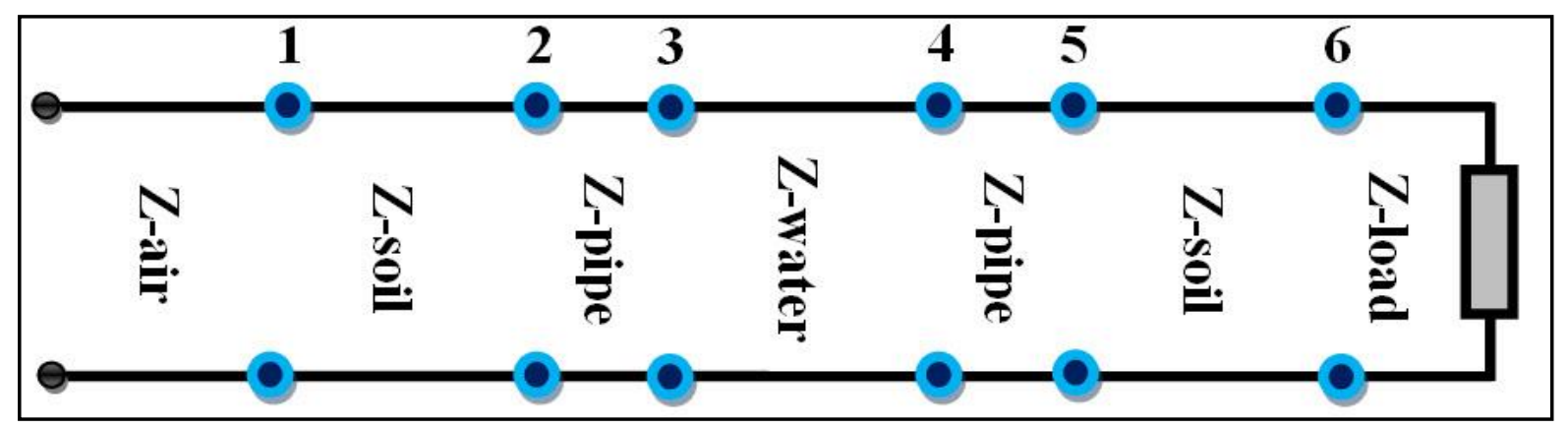

Fig 2. An analogous transmission line with load model 
The multi-layer model equivalent to a transmission line model [12] is used to calculate the total reflection coefficient for water pollution detection model presented in Fig.1. In each layer, the ABCD matrix is calculated and the total reflection coefficient $R_{s}$ from the multi-layer media given by:

$$
R_{s}=\frac{A+B / Z_{s}-Z_{a}\left(C+D / Z_{s}\right)}{A+B / Z_{s}+Z_{a}\left(C+D / Z_{s}\right)}
$$

where

$$
\begin{aligned}
& {\left[\begin{array}{ll}
A & B \\
C & D
\end{array}\right]=\left[\begin{array}{ll}
A_{s} & B_{s} \\
C_{s} & D_{s}
\end{array}\right]\left[\begin{array}{ll}
A_{p} & B_{p} \\
C_{p} & D_{p}
\end{array}\right]\left[\begin{array}{ll}
A_{w} & B_{w} \\
C_{w} & D_{w}
\end{array}\right]\left[\begin{array}{ll}
A_{p} & B_{p} \\
C_{p} & D_{p}
\end{array}\right],} \\
& A_{m}=D_{m}=\cos \left(q_{m} l_{m}\right), B_{m}=j Z_{m} \sin \left(q_{m} l_{m}\right), C_{m}=j \sin \left(q_{m} l_{m}\right) / Z_{m}
\end{aligned}
$$

and $l_{m}$ is the thickness of the $m^{\text {th }}$ layer. The subscript $(a)$ denotes air, the subscript $(s)$ denotes soil, the subscript $(p)$ denotes pipe and the subscript $(w)$ denotes water. The intrinsic impedance of the $m^{\text {th }}$ transmission line $Z_{m}$ is given by:

$$
\mathrm{Z}_{\mathrm{m}}=\sqrt{\frac{\mathrm{j} \omega \mu_{\mathrm{m}}}{\sigma_{\mathrm{m}}+\mathrm{j} \omega \varepsilon_{\mathrm{m}}^{\prime}}}
$$

where $\mu_{m}$ is the permeability and $\sigma_{m}$ is the conductivity. The propagation constant $q_{m}$ is given by:

$$
q_{m}=\beta_{m}-j \alpha_{m}
$$

where $\beta_{m}$ is the phase constant and $\alpha_{m}$ is the attenuation constant of the $m^{\text {th }}$ medium.

Consider a GPR producing a short pulse of duration $T$ and magnitude $V_{\text {Initial }}$ propagates along the transmission line. At each junction, an incident pulse splits into reflected and transmitted pulses. The magnitude of the pulses after each encounter with each junction is indicated by the $\Gamma$ and $\tau$ (reflection and transmission coefficients) values. The considered pulses are the pulse reflected from junction 3 (pipe-water junction) and the pulse reflected from junction 4 (water-pipe junction); hence the pulse $\left(V_{\text {ref } 3}\right)$ reflected from junction 3 (pipe-water junction) is given by:

$$
V_{\text {ref } 3}=\tau_{a s} \tau_{s p} \Gamma_{p w} \tau_{p s} \tau_{s a} e^{-2 \alpha_{a} l_{a}} e^{-2 \alpha_{s} l_{s}} e^{-2 \alpha_{p} l_{p}} V_{\text {Initial }}
$$

where $\tau_{\mathrm{as}}$ is the air-soil transmission coefficient, $\tau_{\mathrm{sp}}$ is the soil-pipe transmission coefficient, $\Gamma_{\mathrm{pw}}$ is the pipewater reflection coefficient, $\tau_{\mathrm{ps}}$ is the pipe-soil transmission coefficient, $\tau_{\mathrm{sa}}$ is the soil-air transmission coefficient, $\alpha_{\mathrm{a}}$ is attenuation constant of air, $\mathrm{l}_{\mathrm{a}}$ is length of air T.L., $\alpha_{\mathrm{s}}$ is attenuation constant of soil, $\mathrm{l}_{\mathrm{s}}$ is length of soil T.L., $\alpha_{p}$ is attenuation constant of pipe material, and $l_{p}$ is length of pipe T.L; hence the pulse $\left(\mathrm{V}_{\mathrm{ref} 4}\right)$ reflected from junction 4 (water-pipe junction) is given by:

$$
V_{\text {ref } 4}=\tau_{a s} \tau_{s p} \tau_{p w} \Gamma_{w p} \tau_{w p} \tau_{p s} \tau_{s a} e^{-2 \alpha_{a} l_{a}} e^{-2 \alpha_{s} l_{s}} e^{-2 \alpha_{p} l_{p}} e^{-2 \alpha_{w} l_{w}} V_{\text {Initial }}
$$

where $\tau_{\mathrm{pw}}$ is the pipe-water transmission coefficient, $\Gamma_{\mathrm{wp}}$ is the water-pipe reflection coefficient, $\tau_{\mathrm{wp}}$ is the water-pipe transmission coefficient, $\alpha_{\mathrm{w}}$ is attenuation constant of water and $\mathrm{l}_{\mathrm{w}}$ is length of water T.L. The reflected signals from upper and lower water edges for pure and polluted water are converted to reflected coefficients $\left(\mathrm{S}_{11}\right)_{\text {ref3 }}$ and $\left(\mathrm{S}_{11}\right)_{\text {ref4 }}$ respectively using the following equation: 


$$
\left(S_{11}\right)_{\text {ref }}=20 \log _{10}\left(\frac{V_{\text {ref }}}{V_{\text {Initial }}}\right)
$$

\section{Dielectric Modeling of Mediums}

Propagation of electromagnetic waves in materials is determined by their electrical parameters, chief amongst these is the permittivity. Permittivity describes the interaction of a material with the electric field and it is a complex quantity:

$$
\varepsilon=\varepsilon^{\prime}-j \varepsilon^{\prime \prime}
$$

where $\varepsilon^{\prime}=\varepsilon_{0} \varepsilon_{r}^{\prime}$ is the dielectric constant, $\varepsilon_{0}$ the free space permittivity, $\varepsilon_{r}^{\prime}$ the relative dielectric constant, $\varepsilon^{\prime \prime}=\varepsilon_{0} \varepsilon_{r}^{\prime \prime}$ is the dielectric loss factor and $\varepsilon_{r}^{\prime \prime}$ the relative dielectric loss factor of the dielectric.

At microwave frequencies, different measurement techniques can be used for the measurement of the dielectric permittivity. These include transmission line techniques (free-space, waveguide and coaxial), impedance and cavity methods. In the current study, the complex dielectric permittivity of the materials of interest is measured using Dielectric Assessment Kit (DAK). DAK system is based on the open-ended coaxial probe technique which uses advanced algorithms and novel hardware for all applications where high-precision dielectric measurements (permittivity, conductivity and loss tangent) are required. The open ended coaxial probe is a cut off section of $50 \mathrm{ohm}$ transmission line used for contact the measured sample either by touching the probe to the surface of a solid/semi-solid media or by immersing it into a liquid media. The electromagnetic fields at the probe end fringe into the material to be measured and its parameters are determined from the change of the scattering parameter $S_{11}$. The dielectric probe used for measurements are designed for fast, precise and non-destructive measurements in the range from $50 \mathrm{MHz}$ to $10 \mathrm{GHz}$. The probe is connected with a phase and amplitude stable cable to a vector network analyzer (VNA) which is then calibrated with open (air), short and liquids with well-known parameters. All calibrations and measurements have been conducted in the closed laboratory facility, environment temperature $25^{\circ} \mathrm{C}$ and humidity $<70 \%$. DAK interfaces with a VNA to measure the scattering parameters seen at the end of the open-ended coaxial probe, while DAK software on an external computer calculates the dielectric constant from the measurement. The measured sample volume is large enough to ensure that reflections at the sample boundaries do not significantly influence the measurements. The minimum sample volume depends on the frequency, probe size and dielectric parameters. DAK measurement system setup is shown in Fig. 3.

Cole-Cole relaxation model is used to fit the measured permittivity of the materials of interest at microwave frequencies; hence the dielectric spectrum can be described by Cole-Cole function [13]:

$$
\varepsilon(v)=\varepsilon_{\infty}+\frac{\varepsilon_{0}-\varepsilon_{\infty}}{1+(i \omega \tau)^{1-\alpha}}-j \frac{\sigma}{\omega \varepsilon_{0}}
$$

where $\varepsilon_{\infty}$ is static permittivity, $\varepsilon_{0}$ is the extrapolated high frequency permittivity, $\tau$ is the discrete relaxation time, and $\omega=2 \pi \nu$, where $\nu$ denotes the frequency, $\sigma$ is the conductivity and $\alpha$ is an exponent factor. A curve fitting routine is implemented based on the least square method to search for the most appropriate values of Cole-Cole fitting model parameters. The electromagnetic model for the scenario presented in Fig. 1 needs a dielectric modelling of materials of interest. 


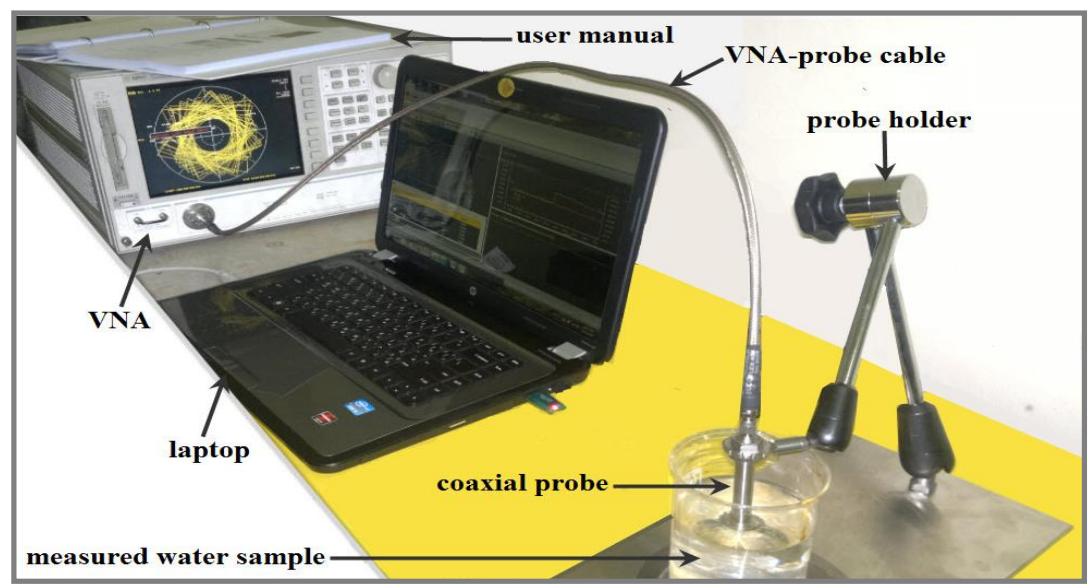

Fig 3. DAK measurement system setup

\subsection{Pure Water}

The dielectric permittivity of pure water is measured at $25^{\circ} \mathrm{C}$ using DAK system and compared with the experimental data done by Kaatze [14]. The best fit sets of parameters of Cole-Cole model for both measurements are tabulated in Table 1. The results of the current study show a reasonable agreement with Kaatze's data and the variability in tabulated data could be interpreted as variations in the used water sample.

Table 1. Cole-Cole model parameters for pure water

\begin{tabular}{cccccc}
\hline parameter & $\boldsymbol{\varepsilon}_{\infty}$ & $\varepsilon_{\mathbf{0}}$ & $\boldsymbol{\tau}(\mathbf{p s})$ & $\boldsymbol{\alpha}$ & $\boldsymbol{\sigma}$ \\
\hline Kaatze's fit & 5.20 & 78.36 & 8.27 & 0.0 & 0.0 \\
Current study & 5.43 & 78.52 & 9.45 & 0.0 & 0.0 \\
\hline
\end{tabular}

\subsection{Polluted Water}

The sampled polluted water is collected from Lake Qarun in the Fayoum of Egypt. The concentrations of pollutants in this water are tabulated in Table 2; these measurements are performed by The Egyptian Central Laboratory of Pools and Water Plants.

Table 2. Pollutants concentrations in polluted water

\begin{tabular}{cccc}
\hline substance & $\begin{array}{c}\text { concentration } \\
(\mathrm{gm} / \mathrm{l})\end{array}$ & substance & $\begin{array}{c}\text { concentration } \\
(\mathrm{gm} / \mathrm{l})\end{array}$ \\
\hline total & & chloride & 13.11 \\
dissolved salts & 36.011 & calcium & 0.499 \\
carbonates & 0.030 & magnesium & 1.377 \\
bicarbonates & 0.036 & sodium & 10.206 \\
sulphates & 9.949 & other ions & 0.534 \\
\hline
\end{tabular}

Polluted water permittivity measurements using the DAK system can be fitted nicely by Cole-Cole function. The best fit sets of parameters of a Cole-Cole model for polluted water are tabulated in Table 3. 
Table 3. Cole-Cole model parameters for polluted water

\begin{tabular}{cccccc}
\hline parameter & $\varepsilon_{\infty}$ & $\varepsilon_{0}$ & $\tau(\mathbf{p s})$ & $\alpha$ & $\sigma$ \\
\hline value & 1.14 & 73.38 & 10.21 & 0.0 & 3.95 \\
\hline
\end{tabular}

The dielectric constant and the dielectric loss factor of pure and polluted water at $25^{\circ} \mathrm{C}$ are compared in Fig. 4. The measured permittivity of pure and polluted water indicate a quite decrease in the dielectric constant and a large increase in the dielectric loss factor of polluted water compared to pure water measurements. This variability in measurements could be interpreted due to the existence of different types of pollutants in polluted water which cause this effect. The effect of pollutants on the dielectric permittivity of water was studied by Raveendranath et al. [15].

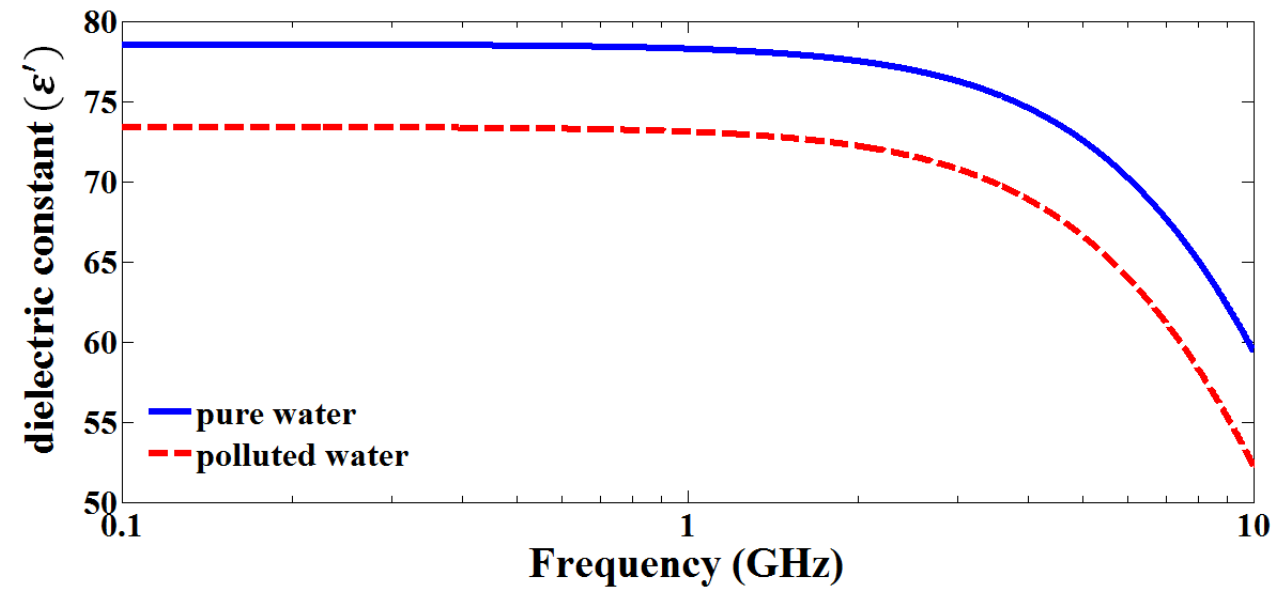

(a)

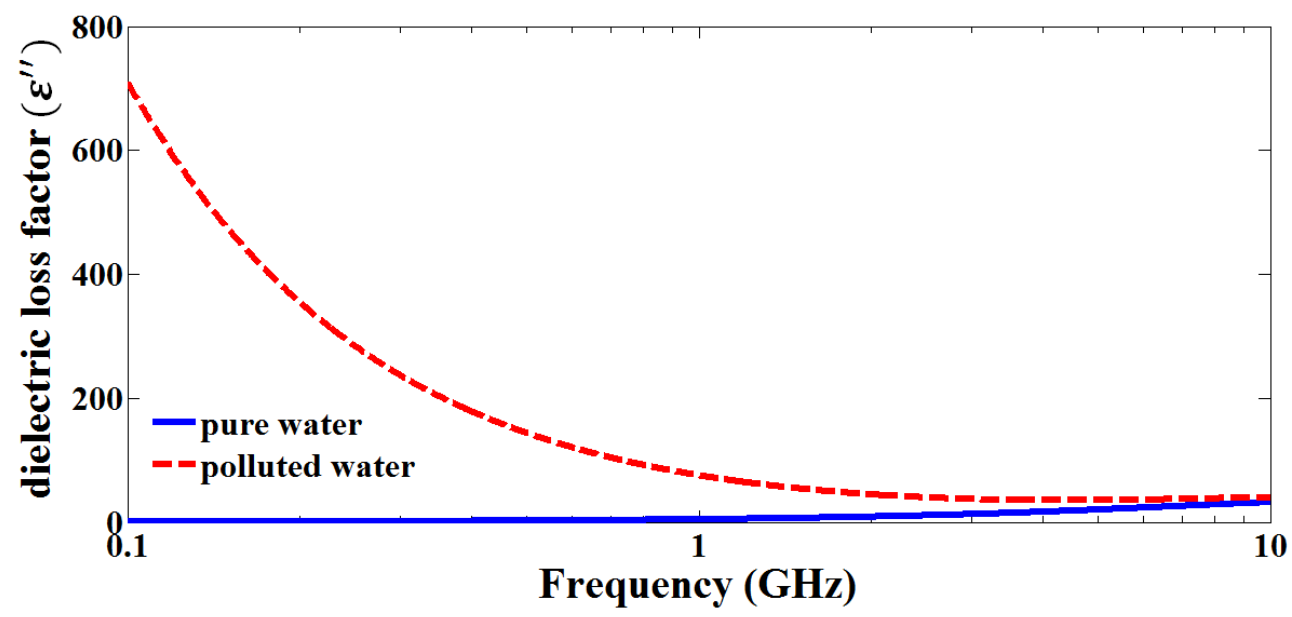

(b)

Fig 4. Measured permittivity of pure and polluted water at room temperature versus frequency [log scale] (a) dielectric constant (b) dielectric loss factor 


\subsection{Soil}

Microwave emission and backscattering of soil depend on its dielectric constant which is effected by the moisture content. Cole-Cole model does not bear direct relationship to the physical properties of soils. The use of dielectric mixing models for soil is advantageous as their parameters are directly related to the soil physical parameters. To predict the performance of electromagnetic sensors it is common to use models that estimate the soil dielectric properties. The relative permittivities of dry and wet soils were measured by Trang [16]. For dry soil with less than $3 \%$ moisture content by weight, the range of $\varepsilon_{\mathrm{r}}^{\prime}$ was from 2.4 to 2.8 , and the range of loss $\operatorname{tangent}\left(\tan \delta=\varepsilon_{\mathrm{r}}^{\prime} / \varepsilon_{\mathrm{r}}^{\prime \prime}\right)$ was from 0.0065 to 0.28 . For moist soil with more than $11 \%$ and less than $22 \%$ moisture content, the range of $\varepsilon_{\mathrm{r}}^{\prime}$ was from 9 to 16 , and the range of loss tangent was from 0.4 to 0.9 . Trang's measurements of dry and wet soils were fairly constant at $0.1 \mathrm{GHz}$ to $4 \mathrm{GHz}$ [17]. In our model, the soil layer is assumed to be homogenous for simplicity of modelling.

\subsection{Pipe}

Polyethylene is one of commonly used materials in water service pipelines. Polyethylene has a dielectric constant of 2.3 and a loss tangent below 0.0005 over a range of $50 \mathrm{MHz}$ to $6 \mathrm{GHz}$ [18].The dimensions which are used for the modeling are tabulated in Table 4.

Table 4. Modelling dimensions

\begin{tabular}{cc}
\hline antenna height (air layer thickness) & $1 \mathrm{~m}$ \\
\hline pipe depth (upper soil layer thickness) & $1 \mathrm{~m}$ \\
pipe material thickness (pipe layer thickness) & $1 \mathrm{~cm}$ \\
inner diameter of pipe (water layer thickness) & $10 \mathrm{~cm}$ \\
\hline
\end{tabular}

\section{Results and Discussion}

\subsection{Frequency domain simulations}

The reflection back from the multi-layer media is simulated based on the dielectric models and the multilayer transmission line model explained in the previous sections. The frequency response is simulated using MATLAB in the range from $100 \mathrm{MHz}$ to $1 \mathrm{GHz}$ for two configurations, the first configuration is where pure water is placed inside the pipe i.e. water layer presented in Fig.1 has pure water parameters and the second one is where polluted water is placed inside the pipe i.e. water layer in Fig. 1 has polluted water parameters. The frequency response of the reflection coefficients for pure and polluted water models are compared in Fig. 5 for dry soil and moist soil with the dimensions presented in Table 4. The results indicate for dry soil significant variations in the reflection coefficient of pure water model vs. Polluted water model while in case of moist soil the variations are insignificant at the simulated range of frequency due to the high attenuation of moist soil which reduces the reflected signal from water inside the pipe so it is recommended for high moist soils to use a lower range of frequency. For dry soil model, the effect of variation of the thickness of soil, pipe and water layers on the reflection coefficient is studied.

\subsubsection{Effect of variation of pipe depth}

Figure 6 illustrate the effect of variation of depth at which the pipe is buried i.e. the variation of the thickness of the soil layer from $1 \mathrm{~m}$ to $0.5 \mathrm{~m}$ and all other dimensions are the same as in Table 4 . The results indicate that 
while the depth decreases the contrast in reflection coefficient increases because of the decrease of the attenuation of soil layer and increase of the reflected signal form water layer.

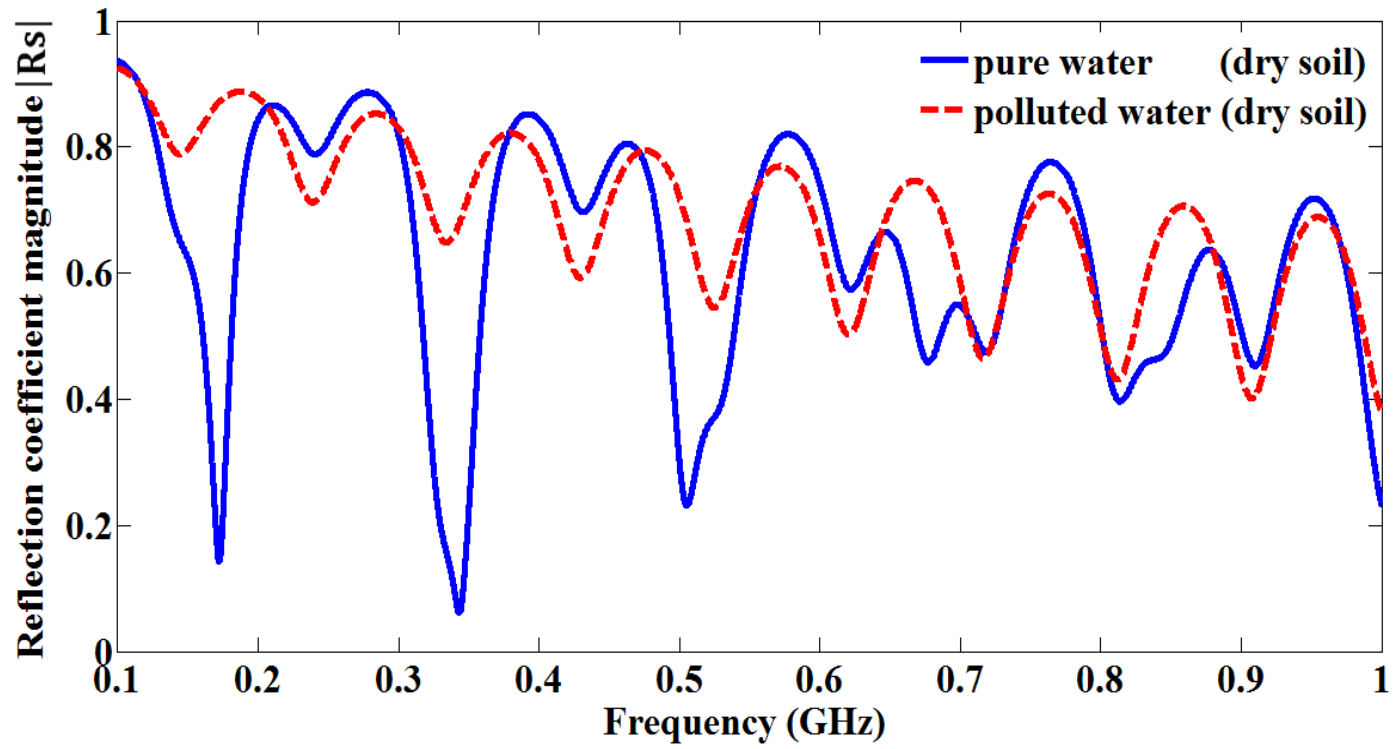

(a)

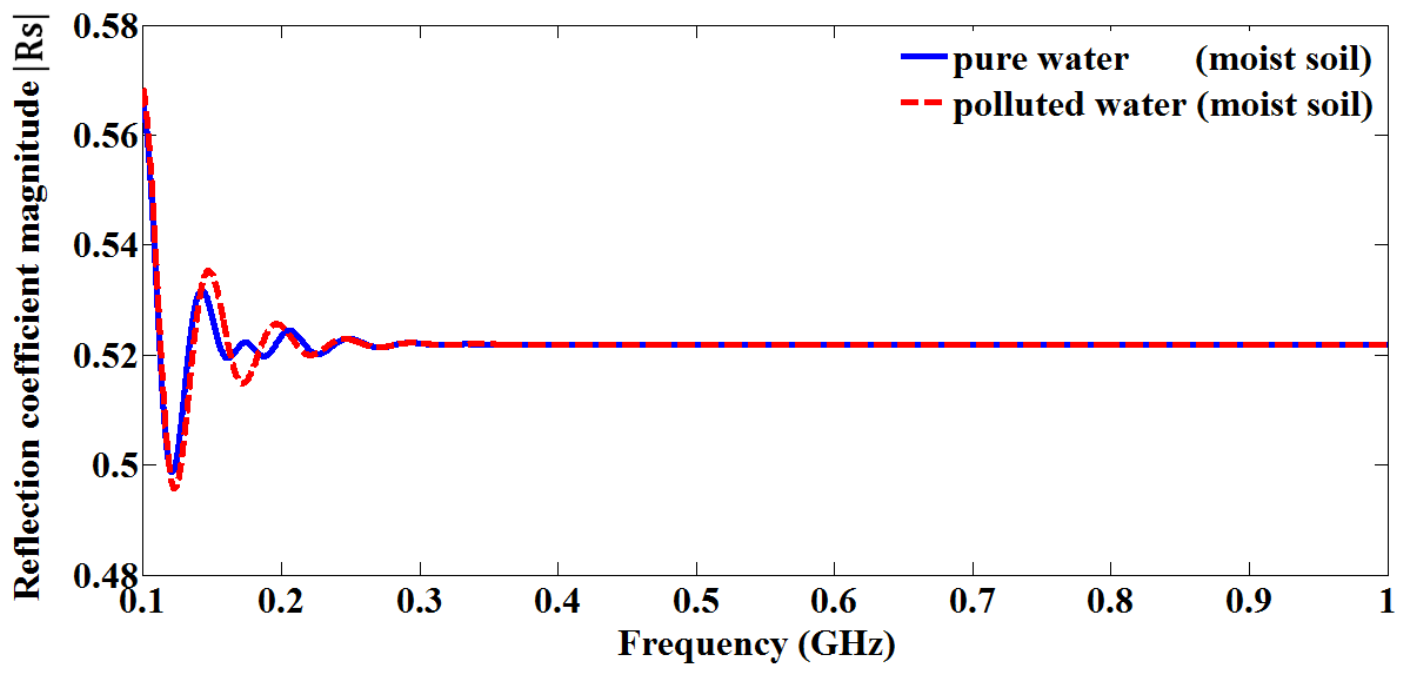

(b)

Fig 5. Frequency response of the absolute value of reflection coefficient for pure and polluted water models (a) dry soil (b) moist soil 


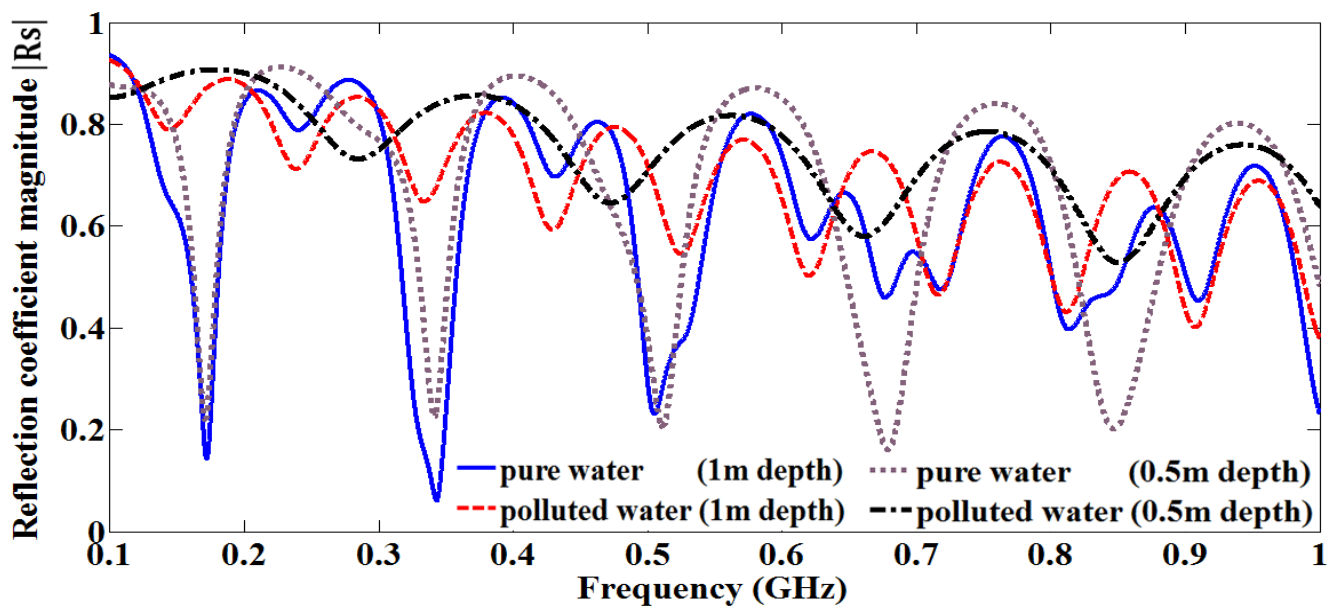

Fig 6. Frequency response of the absolute value of reflection coefficient for pure and polluted water models for dry soil at $1 \mathrm{~m}$ and $0.5 \mathrm{~m}$ depth

\subsubsection{Effect of variation of pipe material thickness}

The effect of the variation of the thickness of the pipe material on the reflection coefficient is studied and the results indicate that the variations in the thickness of the pipe have an insignificant effect on the reflection coefficient.

\subsubsection{Effect of variation of pipe diameter}

The effect of variation of the pipe diameter is also studied and the results indicate that in case of pure water there are variations in the reflection coefficient but in case of polluted water there are insignificant variations in the reflection coefficient. The reflection coefficients of pure and polluted water model are compared in Fig. 7 for a pipe with inner diameter $10 \mathrm{~cm}$ and $20 \mathrm{~cm}$ while all other dimensions are the same as in Table 4 .

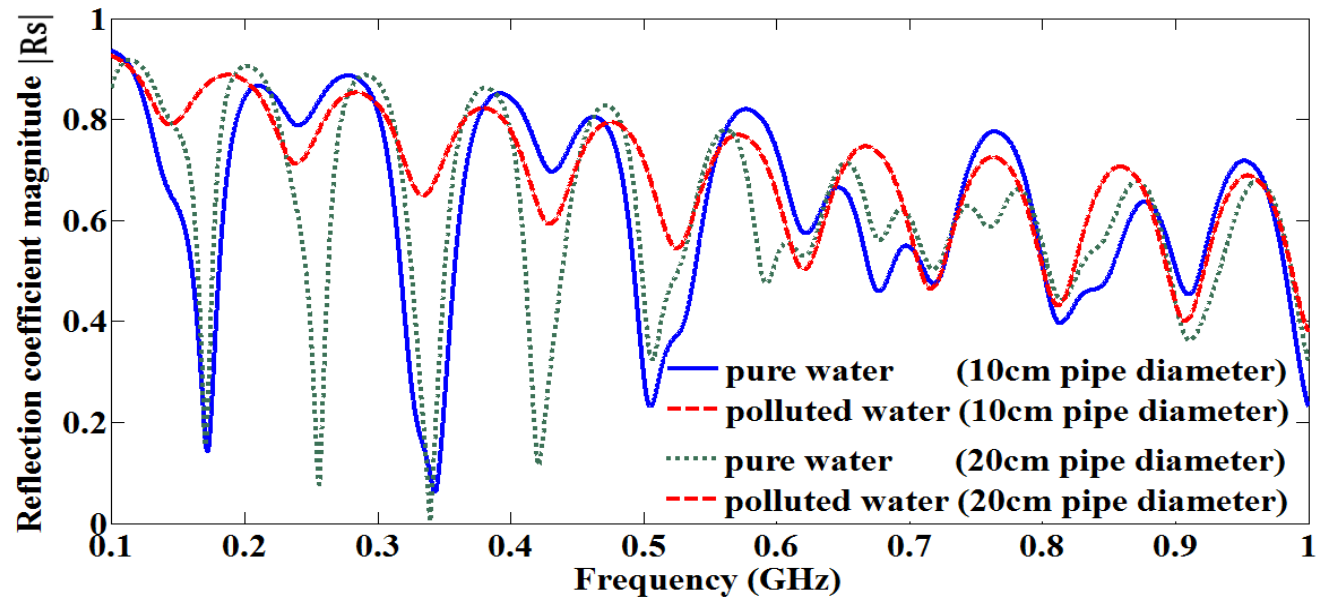

Fig 7. Frequency response of the absolute of reflection coefficient for pure and polluted water models for dry soil with pipe diameter $10 \mathrm{~cm}$ and $20 \mathrm{~cm}$. 
The frequency response of reflected signals from water edges $V_{\text {ref3 }}$ and $V_{\text {ref4 }}$ defined by (4) and (5) respectively of pure and polluted water are compared in Fig. 8 where the initial voltage $V_{\text {Initial }}$ is equal to unity. It is clear from results that the reflected signals form water edges decrease with the increase of frequency due to the increase of attenuation of mediums with the increase of frequency. The small value of reflected signal from the lower edge of polluted water is due to the high signal attenuation caused by the high conductivity of polluted water.

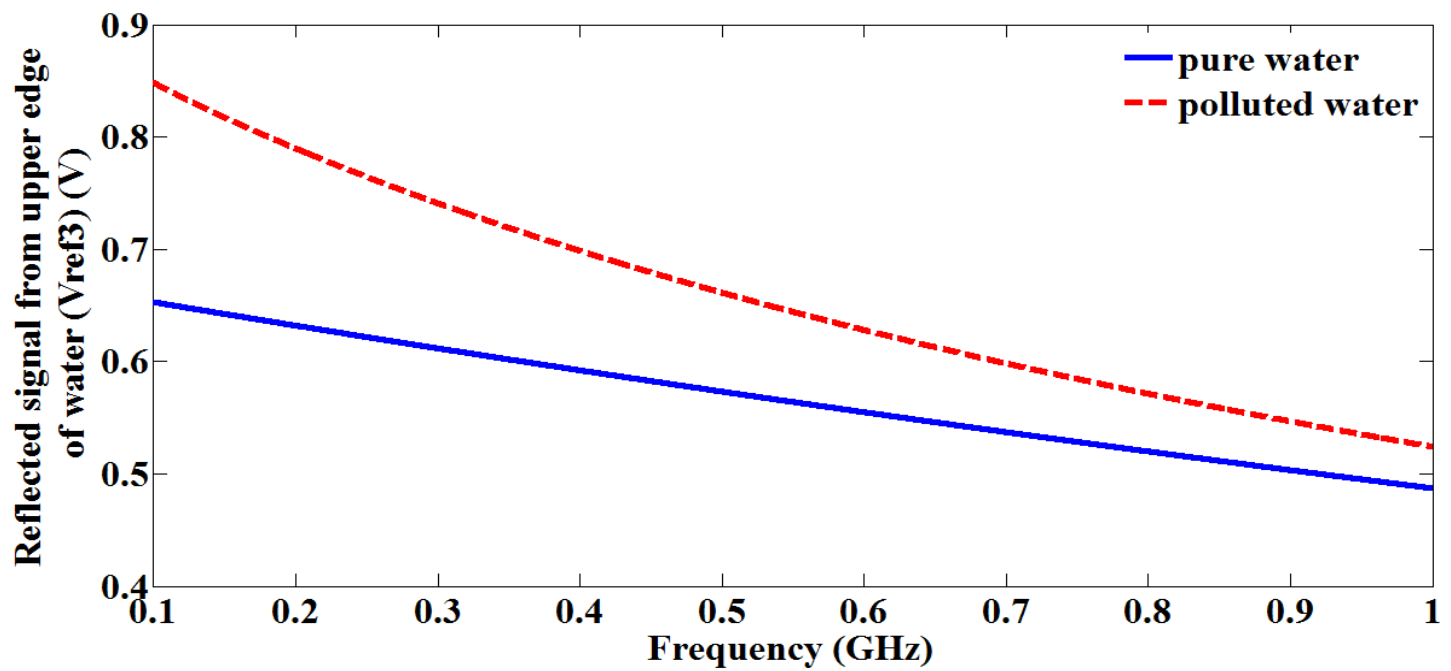

(a)

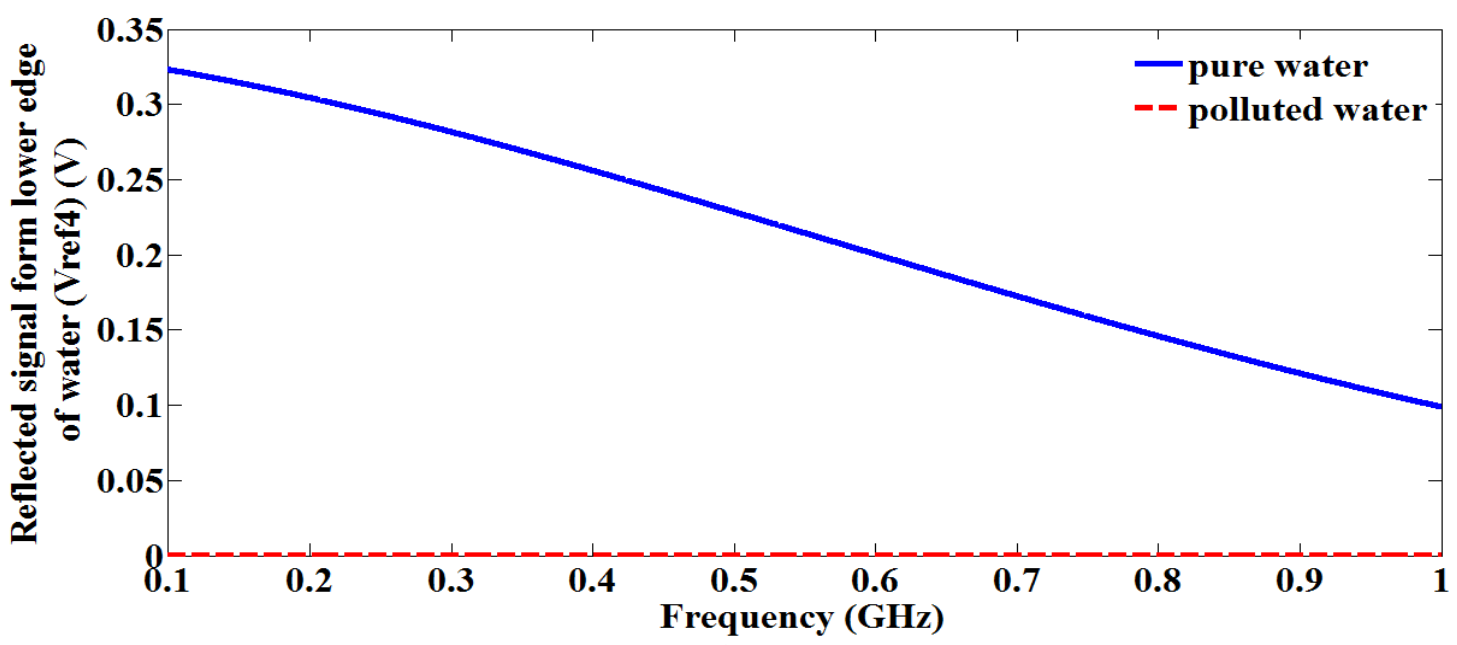

(b)

Fig 8. Frequency response of reflected signal for pure water and polluted water from (a) upper edge of water (b) lower edge of water

\subsection{Time domain simulations}

Time domain simulations are done using transmission line models at $0.5 \mathrm{GHz}$ in the Pspice 10.3 software package. The multi-layer medium is modelled using conventional electrical simulation tools. A $2 \mathrm{~ns}$ sinusoidal 
pulse is used as a transmitted pulse. The voltage wave at the sending end (Antenna) of pure and polluted water models versus time are shown in Fig. 9.

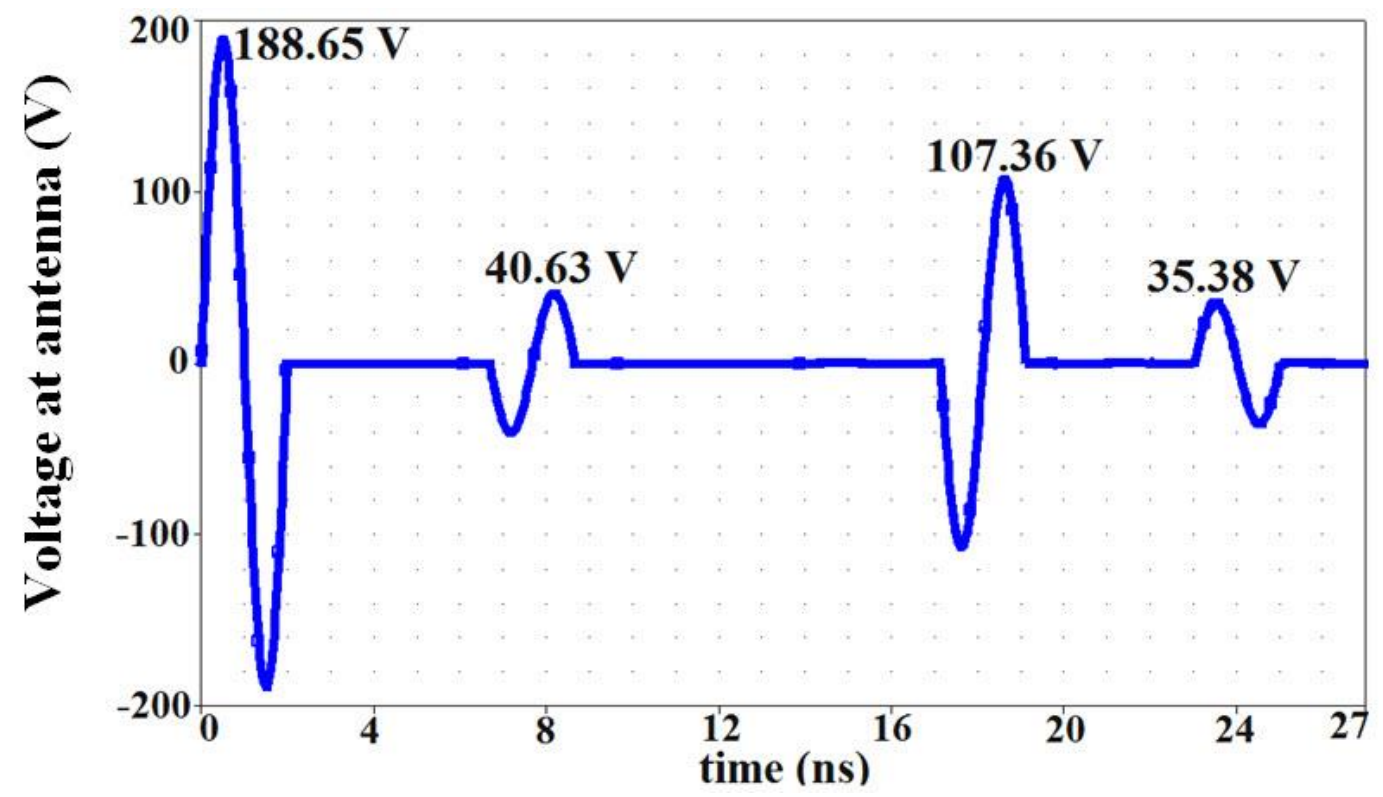

(a)

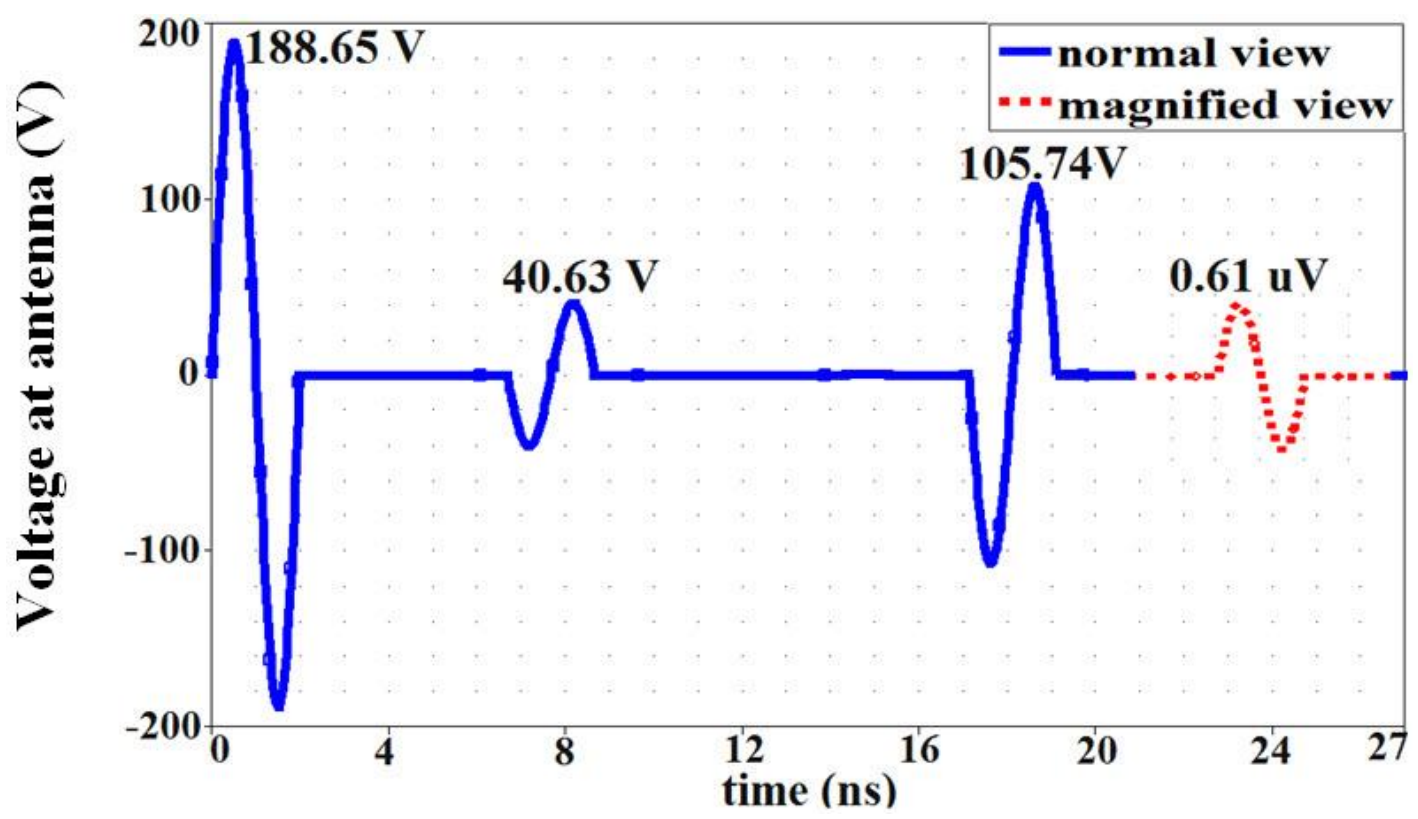

(b)

Fig 9. Voltage at the antenna for (a) pure water model (b) polluted water model 
In the simulation results presented in Fig. 9, the first pulse appears at 0ns, is the transmitted pulse with peak amplitude value $188.65 \mathrm{~V}$. The second pulse is the reflected pulse from air-soill junction. In simulation it appears at $6.67 \mathrm{~ns}$ with a peak amplitude value $40.63 \mathrm{~V}$. The third pulse reflected from soil1-pipe junction pulse is not clearly observed because of its small value due to the small value of soil-pipe reflection coefficient $\Gamma_{s p}$. The considered pulses are the fourth and fifth pulses which are reflected from pipe1-water $v_{r e f 3}$ and waterpipe $2 v_{\text {ref } 4}$ junctions (i.e. water edges) their values are varying according to the type of water in the pipe. The results of reflected coefficients obtained from Pspice simulations and by (4), (5) and (6) are tabulated in Table 5. The differences between simulated and calculated results could be interpreted due to the approximations done by Pspice simulator.

Table 5. The reflected coefficients from water edges obtained by Pspice simulations and by Matlab calculations.

\begin{tabular}{ccccc}
\hline & \multicolumn{2}{c}{$\left(\boldsymbol{S}_{11}\right)_{\text {ref3 }}(\boldsymbol{d B})$} & \multicolumn{2}{c}{$\left(\boldsymbol{S}_{11}\right)_{\text {ref4 }}(\boldsymbol{d B})$} \\
& simulated & calculated & simulated & calculated \\
\hline pure water & -4.90 & -4.83 & -14.54 & -14.60 \\
polluted water & -5.03 & -3.56 & -169.79 & -170.87 \\
\hline
\end{tabular}

The simulation results illustrate that the reflected signals from water edges change if the water is polluted. This change is quite small in the reflected signal of the first edge of water while it is clearly large in the reflected signal of the second edge of water due to the high signal attenuation of polluted water caused by its high conductivity.

\section{Conclusions}

Frequency and time domain behaviours of transmission line models are used to distinguish between polluted and unpolluted water in underground pipelines. The current study shows that there are notable differences in the total reflection coefficients and reflected pulses from water edges for both types of water which could be used in detecting the pollution. The experimental results show that the microwave technique employed in this investigation is an effective method for detecting the presence of pollutants in water. High frequency electromagnetic measurements can prove to be useful tools for monitoring water pollution in underground pipelines.

\section{Acknowledgments}

The authors are thankful to Dr. T. Abou-Elnaga, Electronics Research Institute, for providing the necessary laboratory facilities for measurements.

\section{References}

[1] S. M. Haslam, River Pollution - An Ecological Perspective, Belhaven Press, CBS Pub., Delhi, India, 1991.

[2] P. Niquette, P. Servais and R. Savoir, "Impacts of pipe materials on densities of fixed bacterial biomass in a drinking water distribution system," Wat. Res, vol. 34, No. 6, pp. 1952-1956, 2000.

[3] S. Eyuboglu, H. H. Mahdi and H. J. Al-Shukri, "Detection of water leaks using ground penetrating radar," The 3rd International Conference on Applied Geophysics, 2003, Orlando, America.

[4] N. Al-Dasoqi, A. Mason, R. Alkhaddar, A. Al-Shamma'a, "Use of sensors in wastewater quality monitoring - a review of available technologies, in: I. R. Edward Beighley, W.K. Mark (Eds.) World Environmental and Water Resources Congress 2011: Bearing Knowledge for Sustainability, ASCE, pp. 
354, 2011.

[5] F. Soldovieri, L. Crocco, A. Brancaccio, R. Solimene, R. Persico, "Application of ground penetrating radar and microwave tomography in water monitoring and management," International Water Technology Journal, IWTJ. vol. I, Issue 1, 2011.

[6] S. Lambot, E. C. Slob, D. Chavarro, M. Lubczynski, and H. Vereecken, "Measuring soil surface water content in irrigated areas of southern Tunisia using full-wave inversion of proximal GPR data," Near Surface Geophysics, vol. 16, pp. 403-410, 2008.

[7] S. Jaruwatanadilok, Y. Kuga and A. Ishimaru, "An electromagnetic model for plastic composite materials under obscuring layers," IEEE Antennas and Propagation Society, International Symposium, 2006, pp. 4841-4844, USA.

[8] F. Soldovieri, L. Crocco, A. Brancaccio, R. Solimene and R. Persico, "Applications of ground penetrating radar and microwave tomography in water monitoring and managements," International Water Technology J., vol. I, No. 1, pp. 73-82, 2011.

[9] P. A. Torrione, K. D. Morton, R. Sakaguchi and L. M. Collins, "Histograms of Oriented Gradients for Landmine Detection in Ground-Penetrating Radar Data," IEEE Trans. Geosci. Remote Sens., vol. 52, no. 3, pp. 1539-1550, 2014.

[10] F. Soldovieri, O. Lopera, and S. Lambot, "Combination of advanced inversion techniques for an accurate target localization via GPR for demining applications," IEEE Trans. Geosci. Remote Sens., vol. 49, no. 1, pp. 451-461, 2011.

[11] A. B. Yoldemir and M. Sezgin, "A least squares approach to buried object detection using ground penetrating radar,” IEEE Sens. J., vol. 11, no. 6, pp. 1337-1341, 2011.

[12] Ishimaru, Electromagnetic Wave Propagation, Radiation, and Scattering, Prentice Hall, New York, 1991.

[13] K. S. Cole and R. H. Cole, "Dispersion and absorption in dielectrics I. Alternating current characteristics," Journal of Chemical Physics, vol. 9, pp.341-351, 1941.

[14] U. Kaatze, "The complex permittivity of water as a function of frequency and temperature," J. Chem. Eng. Data, vol. 34, pp. 371-374, 1989.

[15] U. Raveendranath and K.T. Mathew, "Microwave technique for water pollution study," J. of Microwave Power and Electromagnetic Energy, vol. 30, No. 3, pp. 187-195, 1995.

[16] A. H. Trang, "A reference of electromagnetic parameters \& propagation of waves," Notes, pp. 40-49, 1993.

[17] H. Trang, "Simulation of mine detection over dry soil, snow, ice and water," SPIE, vol. 2765, pp. 430-440, 1996.

[18] W. S. Bigelow and E. G. Farr, "Impulse propagation measurements of the dielectric properties of several polymer resins," Farr Research, Inc., Measurement Note 55, 1999.

\section{Authors' Profiles}

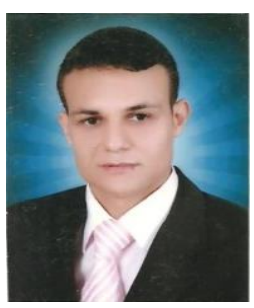

Ahmad H. Abdelgwad was born in Fayoum, Egypt, in 1987. He received the B.S. degree in Electrical Engineering from The University of Fayoum, Fayoum, Egypt, in 2009. He was a Radio Network Engineer with Etisalat Company, Egypt, from 2009 to 2012. He is currently a Demonstrator with Fayoum University with research focused on remote sensing and microwave applications. 


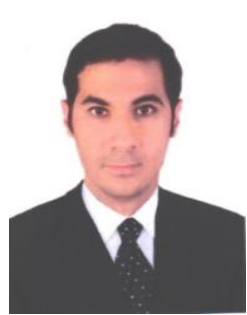

Tarek M. Said was born in Fayoum, Egypt, in 1976. He received the B.S. degree in 1998, M.S. degree in 2004, both in Electrical Engineering from Cairo University, Egypt. In 2009, he received the Ph.D. degree from University of Arkansas at USA. Since 2010, he has joined the Department of Electrical Engineering at Majmaah University in Saudi Arabia. In June 2012, he has been with the Department of Electrical Engineering at Fayoum University, Egypt and currently holds the rank of Assistant Professor. His research interests include dielectric properties of human tissues and multiple scattering of waves in random media.

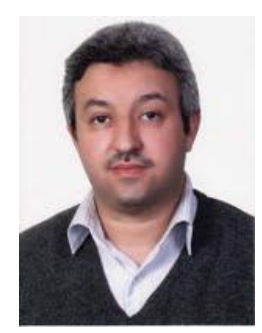

Amr M. Gody received the B.Sc. M.Sc., and PhD. from the Faculty of Engineering, Cairo University, Egypt, in 1991, 1995 and 1999 respectively. He joined the teaching staff of the Electrical Engineering Department, Faculty of Engineering, and Fayoum University, Egypt in 1994. He is author and co-author of about 40 papers in national and international conference proceedings and journals. He is the Acting chief of Electrical Engineering department, Fayoum University in 2010, 2012 till now. His current research areas of interest include speech processing, speech recognition and speech compression.

How to cite this paper: Ahmad H. Abdelgwad, Tarek M. Said, Amr M. Gody,"Microwave Detection of Water Pollution in Underground Pipelines", IJWMT, vol.4, no.3, pp.1-15, 2014.DOI: 10.5815/ijwmt.2014.03.01 